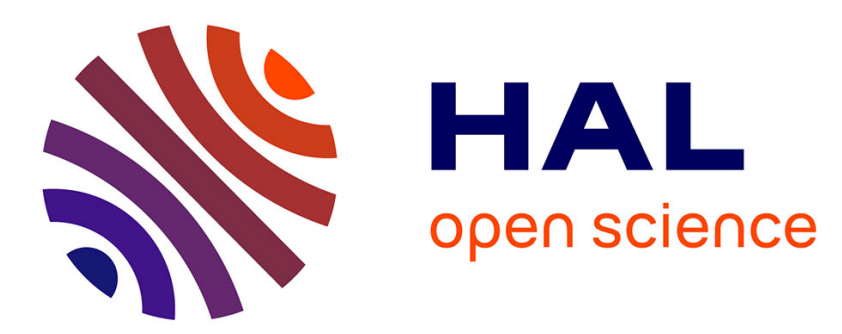

\title{
TRW: An energy storage capacity model for energy harvesting sensors in wireless sensor networks
}

\author{
Junaid A. Khan, Hassaan Khaliq Qureshi, Adnan Iqbal
}

\section{To cite this version:}

Junaid A. Khan, Hassaan Khaliq Qureshi, Adnan Iqbal. TRW: An energy storage capacity model for energy harvesting sensors in wireless sensor networks. IEEE PIMRC 2014, Sep 2014, Washington DC, United States. pp.6, 10.1109/PIMRC.2014.7136487 . hal-01283732

\section{HAL Id: hal-01283732 \\ https://hal.science/hal-01283732}

Submitted on 17 Mar 2016

HAL is a multi-disciplinary open access archive for the deposit and dissemination of scientific research documents, whether they are published or not. The documents may come from teaching and research institutions in France or abroad, or from public or private research centers.
L'archive ouverte pluridisciplinaire HAL, est destinée au dépôt et à la diffusion de documents scientifiques de niveau recherche, publiés ou non, émanant des établissements d'enseignement et de recherche français ou étrangers, des laboratoires publics ou privés. 


\title{
TRW: An Energy Storage Capacity Model for Energy Harvesting Sensors in Wireless Sensor Networks
}

\author{
Junaid Ahmed Khan*† Hassaan Khaliq Qureshi ${ }^{\dagger}$ and Adnan Iqbal, ${ }^{\ddagger}$ \\ * LIGM, University Paris-Est, Marne-la-Vallée, \\ L3i, University of La Rochelle, La Rochelle, France \\ ${ }^{\dagger}$ School of Electrical Engineering and Computer Science \\ National University Of Sciences and Technology, Islamabad, Pakistan \\ $\ddagger$ Namal College, Mianwali, Pakistan \\ *junaid-ahmed.khan@ univ-mlv.fr, ${ }^{\dagger}$ hassaan.khaliq@ seecs.edu.pk, ${ }^{\ddagger}$ adnan.iqbal@ namal.edu.pk
}

\begin{abstract}
Energy provisioning trend in Wireless Sensor Networks (WSNs) is shifted towards alternate sources by utilizing available ambient energy, of which solar irradiance harvesting is considered a viable alternative to fixed batteries. However, the energy storage buffer for harvested solar energy should be adaptive to the sporadic nature of the diurnal solar radiation availability. We believe that the typical fixed battery models no longer apply in harvesting enabled sensors. Therefore, we propose a random walk based stochastic model namely; Trinomial Random Walk (TRW) model for the storage capacity of harvesting enabled sensors. We then apply the proposed model on a comprehensive solar radiation data set of four different locations around the globe. Our performance evaluation demonstrates that the proposed model better analyze the sporadic nature of the diurnal solar radiation availability for estimating the required storage capacity. We further investigate an optimal power consumption value for a given energy store size, such that the utilization of harvested energy is maximized and the probability of energy depletion is minimized. For a given energy harvesting scenario, our model better approximates the optimal load with probability of up to a maximum of $98 \%$, compared to a maximum of $37 \%$ for the binomial random walk model.
\end{abstract}

\section{INTRODUCTION}

Wireless Sensor Networks development started with an idea of coupling energy required by a sensor node since sensorial information exists by enlarge in areas where it is infeasible to provide wired connectivity. Commercially available sensor nodes today are usually powered by typical AA/AAA batteries [1]. However, the limitations of fixed battery design of sensor nodes were soon realized and the research focus quickly shifted towards efficient energy consumption [2, 3]. Energy-efficient design of a sensor hardware, software, algorithms and protocols have served well, but they eventually surrender when the attached batteries are drained. For instance, an energy-efficient protocol may rely on duty cycling of spatio-temporal sensing activities, resulting into application performance degradation for the sake of longer network lifetime. Therefore, it is better to renew energy rather than relying on fixed energy already coupled with the node at the time of network deployment.

Several approaches have been proposed recently including battery replacement [4], energy harvesting from ambient environment $[5,6,7]$ and energy transference from nodes having surplus energy to energy scarce nodes [8,9].

Battery replacement in sensor networks have proven to be difficult and impossible at times, whereas energy-transference from one node to another is a very recent phenomenon. Harvesting energy from ambient environment is being studied for quiet some time and is becoming more wide spread. Ambient energy is available in different forms like solar radiation, wind, ambulatory movement and industrial vibrations [10]. Among all these sources, solar energy is the most powerful resource - estimated to provide $1.4 \mathrm{~kW} / \mathrm{m}^{2}$ [11].

Energy harvesting from ambient environment has opened new dimensions which are unable to realize in fixed battery design. At the same time, it has generated some new questions which do not exist in fixed battery design. One such question is to devise a suitable energy store size. Finding suitable energy store size is important for two reasons; achieving perpetuality and reducing the store size, since increasing storage will increase the cost and size of the node. The answer lies in the analysis of application's energy requirements, energy availability patterns and the ability to harvest energy. Fixed battery design does not make use of available ambient energy, therefore, such models are unfeasible for energy harvesting sensors.

In this paper, we consider this question while considering solar radiation as the ambient energy source. We analyzed historical global solar radiation data from four different locations [12] of the World to provide a storage capacity model to help design an energy efficient algorithm for harvesting enabled sensors. The major contributions of the paper are summarized as follows:

- An energy storage model is presented to characterize the sporadic energy source for harvesting enabled sensors.

- To cater for temporal and spatial changes in energy availability, we have analyzed the proposed model for four different geographically dispersed locations using a comprehensive solar radiation data set.

- To validate the proposed model, comparative analysis of our model with the baseline model is presented. 
- We also consider harvesting enabled sensors with an objective of maximum utilization of the harvested energy without compromising perpertuality. For this purpose, a notion of optimal load is defined, which is the load of a device at which maximum harvesting efficiency is achieved.

Our findings reveal interesting insights in utilizing solar energy in WSNs along with the limitations on the solar energy availability. Trinomial Random Walk model provides better estimates compared to the baseline model in estimating the storage capacity for a harvesting enabled sensor node. It also provides a positive intuition for the feasibility of developing energy harvesting aware algorithms and systems in WSNs due to the small size and low powered sensor nodes.

The rest of this paper is organized as follows. Recent developments in energy harvesting based storage technologies is provided in Section II, Section III explains the TRW model to address the storage requirements. The description of the solar radiation data set is explained in Section IV. Performance evaluation and comparison results are presented in Section V. Finally, Section VI concludes our work along with some insight into the future work.

\section{RELATED WORK}

Ambient energy harvesting is becoming a popular option to overcome the constraints associated with fixed battery design in WSNs. An important metric in harvesting ambient energy for sensor networks is the power harvested per unit area of harvesting circuitry. Solar cells with $44 \%$ efficiency have already been developed, which result into a theoretical maximum of $0.56 \mathrm{~kW} / \mathrm{m}^{2}$ [13]. However, actual efficiency of harvesting devices varies greatly based on available ambient energy - commercial solar panels are optimized for outdoor or indoor usage. Therefore, a harvesting device having high maximum efficiency may perform poorly, if ambient energy is different from required. To solve this problem, Maximum Power Point Tracking (MPPT) techniques is applied [14].

Gorlatova et al. [15] conducted measurements to collect traces for the solar energy availability in indoor environments and proposed algorithms based on deterministic and stochastic energy models. The collected traces are limited in both time duration and area i.e. locations within a campus (indoor) with a maximum time span of upto one year, insufficient for an exhaustive analysis of the solar energy availability.

In [16], the authors proposed a Markov model for multiple source energy harvesting board for body sensors. Based on the remaining energy of the battery, The model predicts the probability that the sensor will detect an event. It poses limitations regarding its dependency on event occurrences as well as the amount of active harvesting boards. Similarly, [17] also provides a model to find the net consumed energy distribution in energy harvesting sensor nodes. However, the energy discharge is dependent on the batteries hardware specifications as well as its limitations to body sensors limits the scope of the model. In Prometheus [6], the intermediate storage for the harvested solar energy is based on super capacitors. Storage hardware such as super-capacitors or batteries limits the analytical models with their varying characteristics regarding the leakage current, charging/discharging efficiencies and degradation over time,
Due to these properties, vital characteristics of the ambient energy source is either overestimated or underestimated. Therefore, motivating the need for a generic analysis of the energy harvesting source behavior.

The Binomial random walk model for solar energy [18] suffers from the lack of dimensions to properly cater the diurnal behavior of the harvested energy. It considers only two possible transitions, an increase in the storage level and a decrease in the storage level by a fixed amount. Beside the increase and decrease in the storage level, we also need to cater for situations when there is no change in the storage level over the course of the day. One such scenario could be when all the daily harvested energy is constantly being utilized by the application and there is no surplus energy to store. In such a case, there is neither energy drawn from the batteries nor does the node gain surplus energy to store in the buffer.

Moreover, it is only able to estimate the size for a large solar array, ignoring key parameters such as small size senors and low power requirements in WSNs. There is a need to study the node's limited energy harvesting ability under different environmental conditions as well as typical energy requirements of a small-sized sensor node. In the next section, we present TRW to address these issues and incorporate an additional degree of freedom required for a harvesting enabled sensor. Unlike previous works, we validated our model against an extensive set of real global solar irradiance traces specifically focusing on harsh environments where frequent batteries replacement is not practical.

\section{TRW: TRINOMIAL RANDOM WALK MODEL}

The storage energy levels can be modeled as a Binomial random walk [18] where transition can occur only between contiguous states, essentially modeling one step movement in a random walk. TRW is an improvement to the Binomial random walk model, in which we incorporate simple albeit vital conditions required to model a storage capacity. These conditions include an increase in the storage capacity, decrease in the storage capacity and the storage capacity retaining its current position. We define three possible transitions with the state transition probabilities $p, q$ and $r$ as shown in Figure 1 . We assume $p$ as the probability of one step increase in the storage state by an amount $\Delta, q$ as the probability of decrease in the storage state by the same amount $\Delta$ and $r$ as the probability of neither decrease nor increase in the storage state, where $\Delta$ is the step size. The relationship $p+q+r=1$ can now be obtained, Now assuming $C=N \cdot \Delta$, as the total storage capacity for a node, where the total storage capacity is divided into $N$ Markov states, each of capacity $\Delta$. By the end

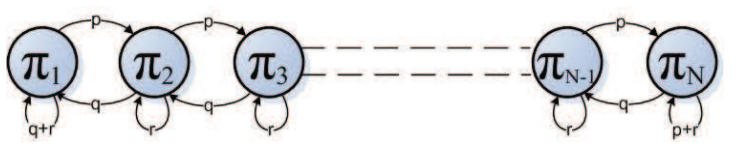

Figure 1: Trinomial Random Walk Model

of a unit time $T$ (divided into small slots $\Delta t$ ), the storage level will be in a state depending on the amount of energy harvested during the previous slots. The steady state probability $\pi_{j}$ of 
occupying the $j^{\text {th }}$ storage state can be expressed as, $\pi_{j}=$ $p \pi_{j-1}+q \pi_{j+1}+r \pi_{j}$. From the transition probabilities between contiguous states $\pi_{j \rightarrow j+1}=p, \pi_{j \rightarrow j-1}=q, \pi_{j \rightarrow j}=r$, and the condition $\pi_{1}+\pi_{2}+\pi_{3}+\ldots \ldots+\pi_{N-1}+\pi_{N}=1$, we generate the transition probability matrix with the following steady state relations:

$$
\begin{aligned}
& \pi_{1}=(q+r) \pi_{1}+q \pi_{2}, \\
& \pi_{2}=p \pi_{1}+r \pi_{2}+q \pi_{3}, \\
& \vdots \\
& \pi_{N-1}=p \pi_{N-2}+r \pi_{N-1}+q \pi_{N}, \\
& \pi_{N}=p \pi_{N-1}+(p+r) \pi_{N},
\end{aligned}
$$

The storage level will be in state $\pi_{1}$ if next unit time period starts with an empty storage, while it will be in state $\pi_{N}$ in the case of fully charged storage. The corresponding steady state probabilities $\pi_{1}$ and $\pi_{N}$ can be finally obtained from the linear equations above:

$$
\begin{aligned}
\pi_{1} & =\frac{(1-\lambda)}{\left(1-\lambda^{N}\right)}, \\
\pi_{N} & =\lambda^{N-1}\left(\pi_{1}\right),
\end{aligned}
$$

where $\lambda=p / q$. For a known capacity $C, \pi_{1}$ and $\pi_{N}$ are the probabilities of being in the lowest storage state and being in the state with maximum energy respectively. Now, the probability of a node to become totally energy deficient is given by the relation $\pi_{E}=(q+r) \cdot \pi_{1}$, Similarly, the probability of a node to have surplus energy is given as $\pi_{S}=(p+r) \cdot \pi_{N}$.

For a prescribed system reliability $\pi_{E}$, we can find the number of required storage states $N$ by inserting $\pi_{1}$ from Equation 1, in the relation $\pi_{E}=(q+r) \cdot \pi_{1}$.

$$
N=\frac{\ln \left[1-\frac{(q+r)(1-\lambda)}{\pi_{E}}\right]}{\ln [\lambda]},
$$

The required number of states $N$ from the above relation with an appropriate step size $\Delta$ provides us the required total storage capacity $C=N \cdot \Delta$, for a sensor node under a given energy deficiency reliability.

\section{Load and probability optimality:}

We are not only interested in keeping the network alive but also desire for the maximum utilization of the harvested energy. Therefore, we extend the model to investigate the optimal load. We define optimal load as the node's optimal power consumption value, at which it utilizes maximum harvested energy. We also define optimal probability $\pi_{o p t}=1-\left(\pi_{1}+\pi_{N}\right)$ as the probability of neither being in the energy depletion state nor in the energy surplus state. We are interested in both extreme probabilities to avoid two situations:

- Wastage of excess daily solar energy: This situation arise when the storage level is at maximum capacity and is unable to accommodate excess harvested energy during the course of the day. This results in wastage of the extra harvested solar energy due to underestimation of the storage energy levels.

- Energy depletion due to low daily solar irradiance: This is a more common situation, when there is not
Table I: Data set specifications

\begin{tabular}{l|l|l|l|l|l|l}
\hline Name & Coordinates & $\begin{array}{l}\text { Mean } \\
\text { Annual } \\
\text { Temp }\end{array}$ & $\begin{array}{l}\text { Data } \\
\text { (days) }\end{array}$ & $\begin{array}{l}\text { Mean } \\
\text { Burst } \\
\text { Size }\end{array}$ & $\begin{array}{l}\text { Max } \\
\text { Burst } \\
\text { Size }\end{array}$ & $\begin{array}{l}\text { Mean Daily } \\
\text { Radiation } \\
\left(K W / m^{2}\right)\end{array}$ \\
\hline $\begin{array}{l}\text { Tamanrasset, } \\
\text { Algeria } \\
\begin{array}{l}\text { Valentia, } \\
\text { Ireland }\end{array}\end{array}$ & $22.78 \mathrm{~N}, 5.52 \mathrm{E}$ & $21.1^{\circ} \mathrm{C}$ & 4744 & 1.32 & 06 & 2.28 \\
$\begin{array}{l}\text { Bondville, } \\
\text { USA }\end{array}$ & $40.06 \mathrm{~N}, 10.25 \mathrm{E}, 37 \mathrm{~W}$ & $11.4^{\circ} \mathrm{C}$ & 5752 & 3.85 & 24 & 0.99 \\
$\begin{array}{l}\text { Sonnblick, } \\
\text { Austria }\end{array}$ & $47.05 \mathrm{~N}, 12.95 \mathrm{~W}$ & $-04^{\circ} \mathrm{C}$ & 4080 & 1.39 & 05 & 1.47 \\
\hline
\end{tabular}

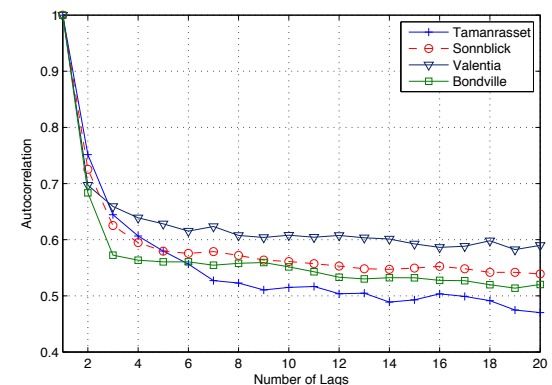

Figure 2: Autocorrelation of solar irradiance unavailability

sufficient solar energy harvested during the course of a day to keep the node operational, thus resulting in energy deficiency at the node, affecting the overall network lifetime.

The worst case scenario (power loss probability) is modeled by the state $\pi_{1}$, the probability of a node to be in the lowest energy state. On the other hand, quantifying the state $\pi_{N}$, provides us the probability of a node to be in the state $N$ (maximum storage capacity), identifying the necessary condition for a node to receive surplus energy and cater for the excess solar energy harvested during a particular day. Any harvesting aware energy management algorithm can adapt to efficiently operate in an Energy Neutral state by avoiding these extreme states, thus, maximizing network lifetime.

\section{DATA-SET DESCRIPTION}

To analyze battery size requirements in different parts of the World for a solar energy harvesting device, we obtained daily solar radiation data set from four different locations, Tamanrasset (Algeria), Valentia (Ireland), Bondville (Illinois, USA) and Sonnblick (Austria). This data is publicly available at World Radiation Data Center (WRDC) [12]. This data set contains solar radiation measurements for many sites in the World. We only considered those sites which provide data for maximum possible number of consecutive days. The minimum number of days in our analysis is 4080 (Bondville) and the maximum is 6936 for Sonnblick. The sites used in this analysis also vary greatly in solar radiation characteristics. For instance, Sonnblick (Austria) has harsh weather conditions for harvesting solar energy, since it has low average temperature, whereas Tamanrasset (Algeria) possesses better opportunity for harvesting as evident from relatively higher average annual temperature of about $21.1^{\circ} \mathrm{C}$. 


\section{Burst of solar radiation unavailability and autocorrelation:}

As the main interest lies in finding the suitable size for a battery storage under solar radiation harvesting, the first crude measure is the expected number of consecutive days when harvested solar energy is less then a certain threshold. We have computed the burst size for each site as the consecutive number of days, in which the daily global solar energy received is less than $0.2 \mathrm{KWh} / \mathrm{m}^{2}$. We chose this threshold assuming that around $0.5 \mathrm{~mW}$ power is required for minimum node operation [1]. Table I shows the maximum and mean burst size for each location. The mean burst size between 1 and 4 days indicates that a variation exists between different sites, hence optimal energy store sizes may not be the same. The variation of the maximum burst size also indicates the same observation.

We further analyzed this data by computing the autocorrelation. Autocorrelation information provides us the analysis of the solar radiation availability dependence between consecutive days. Let the solar radiation received at a discrete time instance $i$ be a binary random process represented as $X[i] \in\{0,1\}$, where 0 , refers to the time slot when the daily received solar radiation is below the minimum threshold value. The autocorrelation measure can be defined as:

$$
\rho[k]=\frac{E\{X[0] X[k]\}-E\{X[0]\} E\{X[k]\}}{\sqrt{\operatorname{var}\{X[0]\}} \sqrt{\operatorname{var}\{X[k]\}}},
$$

where $E\{$.$\} and \operatorname{var}\{$.$\} represent the expected value and$ variance of the random process. From the available data set for an ensemble of days, we compute sample expectations and sample variances of the burst size, which are then used to compute the sample autocorrelation for each data set. Autocorrelation results of up to 20 lags are shown in Figure 2. From these results, we found that the first few lags are strongly correlated, with first one being the most strongly correlated. These results are in line with the burst size analysis, where we found that the mean burst size is between 1 and 4 days.

\section{Performance Evaluation And Results}

We evaluated TRW model using real World solar radiation data set described in the previous section. Our evaluation focuses on the analysis of the global availability of ambient energy rather than being limited to a specific storage medium. Storage medium, either super-capacitors or batteries posses variable properties regarding the leakage current, charging/discharging efficiency and degradation over time. This leads to mis-estimation of the sporadic availability of the ambient source.

Initially we analyzed the availability of solar irradiance during the course of a single instance (i.e. 24hrs) and then widened our analysis to cater for continuous diurnal behavior of a maximum ensemble of up to 19 consecutive years (e.g. for Sonnblick : 6936 days). For a single day, let $A=P_{p k} . \phi$ be the diurnal solar radiation output of a solar panel attached to a node, where $P_{p k}$ is the peak harvesting efficiency and $\phi$ is the daily harvested solar energy. Assume $\mu_{A}$ and $\sigma_{A}$ be the mean and variance of the radiation data. Assuming an average load $L$ at each node including the application consumption as well as the non-ideal storage characteristics. The difference between the source and the load, $D=A-L$, gives us its mean and variance $\mu_{D}$ and $\sigma_{D}$ respectively. We derive the step size
Table II: TRW parameters for active TELOS load $(C=10.08 W h, L=$ $1.41 W h)$

\begin{tabular}{l|c|c|c|r}
\hline Variable & Tamanrasset & Sonnblick & Valentia & Bondville \\
\hline$\mu_{A}$ & 6.175 & 3.931 & 2.663 & 3.980 \\
$\mu_{D}$ & 6.027 & 3.783 & 2.516 & 3.832 \\
$\sigma_{D}$ & 2.035 & 4.454 & 4.050 & 5.094 \\
$\mathrm{p}$ & 0.099 & 0.130 & 0.133 & 0.134 \\
$\mathrm{q}$ & 0.100 & 0.126 & 0.129 & 0.127 \\
$\mathrm{r}$ & 0.800 & 0.745 & 0.738 & 0.740 \\
$\Delta$ & 1.07 & 1.846 & 1.720 & 1.997 \\
$\mathrm{~N}$ & 9.30 & 5.42 & 5.82 & 5.00 \\
$\lambda$ & 0.990 & 1.028 & 1.03 & 1.05 \\
$\pi_{1}$ & 0.100 & 0.174 & 0.160 & 0.170 \\
$\pi_{N}$ & 0.100 & 0.196 & 0.184 & 0.220 \\
$\pi_{\text {opt }}$ & 0.800 & 0.631 & 0.656 & 0.590 \\
\hline
\end{tabular}

$\Delta$ and the transition probabilities $p, q$ and $r$ for our model using the above parameters, providing us the respective steady state probabilities.

According to our knowledge, the existing models are unable to clearly identify the behavior of the diurnal harvested energy independent of the limitations posed by the storage medium, failing to estimate the source energy availability. Therefore, TRW is validated by comparing with the Binomial random walk model in order to validate our assumptions regarding storage state behavior for the daily harvested irradiance solely focusing on the energy source availability independent of the storage medium. In the following sections, we describe the results of applying both models on the real solar radiation data. We assumed that the maximum efficiency of the solar panel is under $44 \%$ as indicated by the latest research in solar energy [13]. The size of the solar panel is considered as $5 \times 5 \mathrm{~cm}^{2}$ for a TelosB mote under consideration. For the first set of results, we consider the active load of a TelosB mote [1], since TelosB hardware design provides an optimal power consumption [19]. We also assumed the maximum available storage capacity of up to four typical AA batteries $10.08 \mathrm{Wh}$. The parameters derived for TRW using the four data set are shown in Table II. We evaluated our model using various load values but here we only show the parameters assuming TelosB active load for brevity, although similar trend is observed by varying load for different commercial motes.

\section{A. Storage state comparison}

The performance benchmark of TRW can be better analyzed by finding how better it follows the real solar radiation trace. Therefore, we generated traces using the storage states information from the real data. We deduce the transition probabilities $p, q$ and $r$ for TRW using first half of the data set. Similarly, we also computed the corresponding transition probabilities $p$ and $q$ for the Binomial random walk model since we need to provide a comparative analysis for both schemes. By starting with an initial storage level $B_{0}$, we validated the generated traces using the first half of the data set for both models keeping the second half of the real data set as baseline.

Figure 3 compares energy storage levels up to one year using the real data for each site mentioned before in Table I as baseline. The horizontal axis shows the number of days against its respective storage state on the vertical axis. We show the trend only for one year on the horizontal axis, however, similar pattern is followed for the entire data set due to the diurnal repetition of solar radiation each year. The Binomial random walk model assumes a necessary transition between two consecutive days and does cater for the situation where 

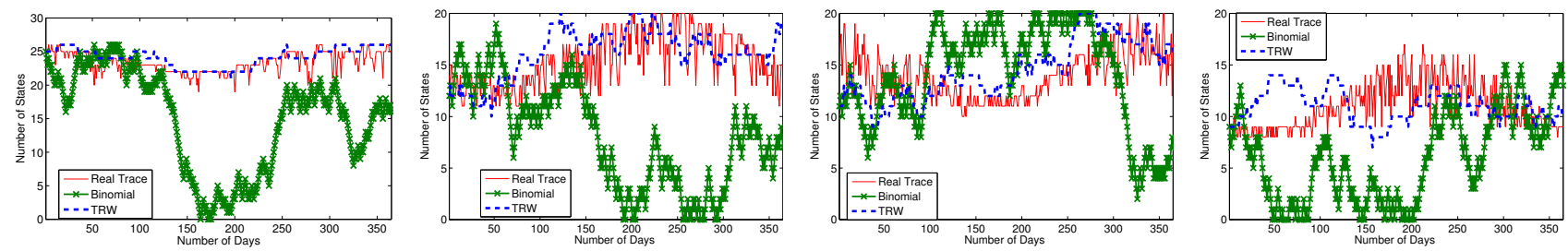

Figure 3: Annual storage state comparison: (Left to Right) Tamanrasset, Bondville, Sonnblick and Valentia
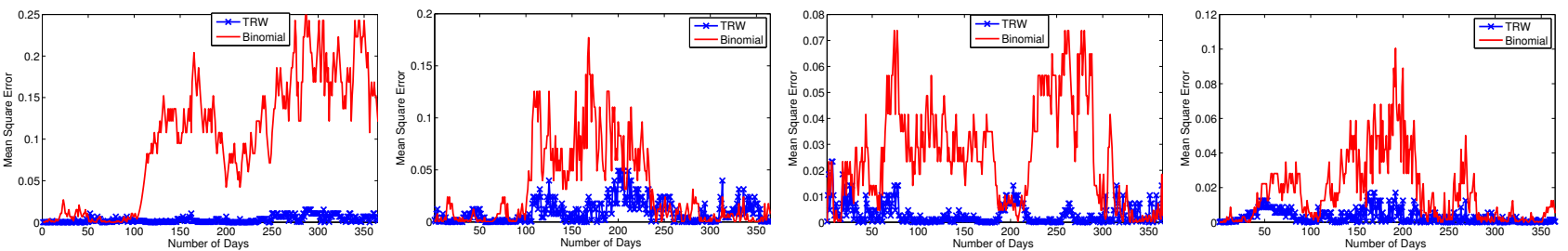

Figure 4: Annual Mean Square Error (MSE) comparison: (Left to Right) Tamanrasset, Bondville, Sonnblick and Valentia

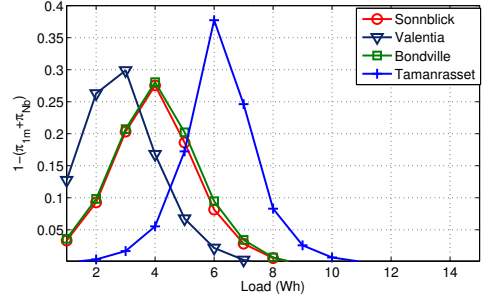

(a) Optimal probability comparison using Binomial random walk for each location with increasing load while keeping capacity fixed to 4 AA batteries

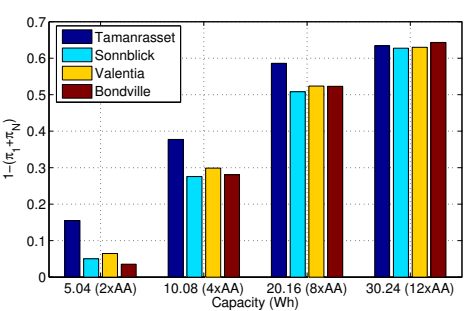

(b) Impact of change in energy store size on optimal probability using Binomial random walk while load remains optimal

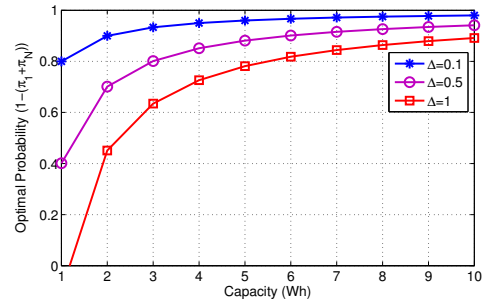

(c) Impact of varying capacity and $\Delta$ on optimal probability estimation using TRW

Figure 5: Optimal Load and Optimal Probabilities comparison

Table III: MSE and Mutual Information

\begin{tabular}{l|l|l|l|l}
\hline \multicolumn{2}{c|}{ Location } & TRW & Binomial & Real Data \\
\hline Tamanrasset, & MSE & 0.0182 & 0.0587 & 0.0097 \\
Algeria & MI & 0.1485 & 0.1021 & 0.2943 \\
\hline Bondville, IL, & MSE & 0.0269 & 0.0541 & 0.0116 \\
USA & MI & 0.1526 & 0.1161 & 0.2354 \\
\hline Sonnblick, & MSE & 0.0077 & 0.0162 & 0.0051 \\
Austria & MI & 0.1192 & 0.0822 & 0.3027 \\
\hline \multirow{2}{*}{ Valentia, Ireland } & MSE & 0.0088 & 0.0116 & 0.0020 \\
& MI & 0.1202 & 0.0486 & 0.2384 \\
\hline
\end{tabular}

the storage state retain its current position, thus, it results in large number of fluctuation between states. These results also verify that despite the irregularities due to the randomness of the diurnal solar irradiance, TRW better follow the real trace for each site due to the state recurring property.

\section{B. MSE and mutual information}

We further validated our model by finding the mean square error (MSE) and the mutual information of each model against the real trace. MSE and mutual information reflects the extent to which both, TRW and the Binomial random walk deviates in modeling the original solar radiation pattern. Table III shows the respective MSE and the mutual information against the real trace for both models. The MSE between TRW and the real trace is significantly less compared to the Binomial random walk model. This can also be verified from the third column which shows the MSE and mutual information of the first half of the real data set with the second half. Similar results can be observed in Figure 4 which depicts the mean square error comparison of both schemes with the real data for each site. The horizontal axis shows the number of days, for which the MSE is computed and the vertical axis shows the MSE. From these MSE plots for all sites, we see that the MSE of TRW with the real data is lesser compared to the Binomial random walk. The mutual information comparison in Table III also validates our claims for the storage capacity of harvesting enabled sensors.

\section{Load and probability optimality comparison}

Sensor applications usually aims to utilize the maximum available energy, therefore, it is important to find the impact of varying the storage energy level on the optimal load and the optimal probability. Therefore, we evaluated the impact of varying the storage energy levels and the step size $\Delta$ on the 
probability of depletion, probability of being in energy surplus state and the corresponding optimal probability for each site. Figure $5 \mathrm{a}$ plots the optimal probability $\pi_{o p t}$ by increasing load using Binomial random walk model for energy storage capacity of $10.08 W h$ ( $4 \times$ AA batteries $)$ for all sites. The peaks indicates the optimal point where the utilization of the harvested energy is maximized. For a storage capacity of upto 4 AA batteries, it limits the maximum supportable load per day to $(6 W h)$ for Tamanrasset, estimating the optimal probability upto only $37 \%$ as shown in the Figure $5 \mathrm{a}$.

Similarly, Figure 5b shows the optimal probabilities estimated using the Binomial random walk model for different set of AA batteries. The horizontal axis shows the set of batteries, while the vertical axis shows the optimal probability. It is shown that low storage capacities results in lower optimal probabilities. On the other hand, Figure 5c shows the optimal probability estimation for a storage capacity for the same storage capacity (4 AA batteries) using TRW. The horizontal axis shows the increase in the capacity, while the vertical axis shows the optimal probability. It clearly highlights that, even for lower values of $\Delta$ and capacity $C$, the TRW model takes useful information into consideration where maximum optimal load can be supported. It also provides better estimation of the optimal probabilities i.e. from the real data, an optimal probability of upto 0.8 can be achieved with $\Delta$ even as lower as 0.1 . It also estimates the optimal probability upto a maximum of $98 \%$ for the same $\Delta$. This can also be cross-validated by the last row of Table II, which shows the optimal probability for each location for a given capacity. This shows that an optimal load for a given energy harvesting situation can be maintained by considering the additional dimension for the storage state.

The comparative analysis of both model concludes that the TRW provide a better estimation of the storage capacity for harvesting enabled sensor deployed in any part of the World. The optimal probability analysis also validates our claim regarding the estimation of the optimal power consumption for a given energy harvesting scenario.

\section{CONClusions AND Future Work}

Harvesting ambient energy for sensors is studied for some time and is becoming more popular. Therefore, we provided a generic model for the energy storage capacity for harvesting enabled sensors. We analyzed our model by applying it on an extensive real World solar radiation data set. We used the data set to analyze the diurnal behavior of the solar irradiance pattern for different global regions for a maximum ensemble of days. This analysis provides an insight into the design of harvesting aware systems and algorithm under different geographical conditions. Additionally, we defined an optimal point where utilization of the harvested energy is maximum for a given energy harvesting scenario. Based on the real data analysis, the proposed model presents a closer fit compared to the Binomial random walk.

Currently, we are extending the model to further improve the storage state accuracy. We are planning to use TRW model to adjust the node's power consumption in accordance with the storage state conditions. Specifically, the sensor node should simultaneously adapt to the availability of ambient energy as well as the residual stored energy.

\section{REFERENCES}

[1] "Memsic wireless modules- http://www.memsic.com/products/ wirelesssensor-networks/wireless-modules.html."

[2] S. Rizvi, H. K. Qureshi, S. Ali Khayam, V. Rakocevic, and M. Rajarajan, "A1: An energy efficient topology control algorithm for connected area coverage in wireless sensor networks," Journal of Network and Computer Applications, vol. 35, no. 2, pp. 597-605, 2012.

[3] G. Anastasi, M. Conti, M. Di Francesco, and A. Passarella, "Energy conservation in wireless sensor networks: A survey," Ad Hoc Networks, vol. 7, no. 3, pp. 537-568, 2009.

[4] B. Tong, Z. Li, G. Wang, and W. Zhang, "On-demand node reclamation and replacement for guaranteed area coverage in long-lived sensor networks," in Quality of Service in Heterogeneous Networks. Springer, 2009, pp. 148-166.

[5] A. Kansal, J. Hsu, S. Zahedi, and M. B. Srivastava, "Power management in energy harvesting sensor networks," ACM Transactions on Embedded Computing Systems (TECS), vol. 6, no. 4, p. 32, 2007.

[6] X. Jiang, J. Polastre, and D. Culler, "Perpetual environmentally powered sensor networks," in Information Processing in Sensor Networks, 2005. IPSN 2005. Fourth International Symposium on. IEEE, 2005, pp. $463-$ 468.

[7] W. K. Seah, Z. A. Eu, and H.-P. Tan, "Wireless sensor networks powered by ambient energy harvesting (wsn-heap)-survey and challenges," in Wireless Communication, Vehicular Technology, Information Theory and Aerospace \& Electronic Systems Technology, 2009. Wireless VITAE 2009. 1st International Conference on. IEEE, 2009, pp. 1-5.

[8] M. K. Watfa, H. Al-Hassanieh, and S. Selman, "Multi-hop wireless energy transfer in wsns," Communications Letters, IEEE, vol. 15, no. 12, pp. 1275-1277, 2011.

[9] L. Xie, Y. Shi, Y. T. Hou, W. Lou, and H. D. Sherali, "On traveling path and related problems for a mobile station in a rechargeable sensor network," in Proceedings of the fourteenth ACM international symposium on Mobile ad hoc networking and computing. ACM, 2013, pp. 109-118.

[10] J. A. Paradiso and T. Starner, "Energy scavenging for mobile and wireless electronics," Pervasive Computing, IEEE, vol. 4, no. 1, pp. 18 27, 2005.

[11] S. Sudevalayam and P. Kulkarni, "Energy harvesting sensor nodes: Survey and implications," Communications Surveys \& Tutorials, IEEE, vol. 13, no. 3, pp. 443-461, 2011.

[12] "World radiation data base-http://wrdc.mgo.rssi.ru/ and nerl solar radiation research laboratory-http://www.nrel.gov/midc/srrl."

[13] "Maximum solar panel efficiency by fraunhofer-institut für solare energiesysteme ise in science daily 2014 ://www.sciencedaily.com/releases/2013/09/130923204214.htm."

[14] S. Moon, S.-J. Kim, J.-W. Seo, J.-H. Park, C. Park, and C.-S. Chung, "Maximum power point tracking without current sensor for photovoltaic module integrated converter using zigbee wireless network," International Journal of Electrical Power \& Energy Systems, vol. 56, pp. 286297, 2014.

[15] M. Gorlatova, A. Wallwater, and G. Zussman, "Networking low-power energy harvesting devices: Measurements and algorithms," Mobile Computing, IEEE Transactions on, vol. 12, no. 9, pp. 1853-1865, 2013.

[16] J. Ventura and K. Chowdhury, "Markov modeling of energy harvesting body sensor networks," in Personal Indoor and Mobile Radio Communications (PIMRC), 2011 IEEE 22nd International Symposium on. IEEE, 2011, pp. 2168-2172.

[17] M. Y. Naderi, S. Basagni, and K. R. Chowdhury, "Modeling the residual energy and lifetime of energy harvesting sensor nodes," in Global Communications Conference (GLOBECOM), 2012 IEEE. IEEE, 2012, pp. 3394-3400.

[18] L. L. Bucciarelli Jr, "Estimating loss-of-power probabilities of standalone photovoltaic solar energy systems," Solar Energy, vol. 32, no. 2, pp. 205-209, 1984.

[19] J. Polastre, R. Szewczyk, and D. Culler, "Telos: enabling ultra-low power wireless research," in Information Processing in Sensor Networks, 2005. IPSN 2005. Fourth International Symposium on. IEEE, 2005, pp. 364369. 